\title{
SIMULATING THE “SLIDING DOORS” EFFECT THROUGH MAGNETIC FLUX EMERGENCE
}

\author{
David MacTaggart and Alan W. Hood \\ School of Mathematics and Statistics, University of St Andrews, St Andrews, Fife KY16 9SS, UK; dm428@st-andrews.ac.uk \\ Received 2010 March 31; accepted 2010 May 26; published 2010 June 4
}

\begin{abstract}
Recent Hinode photospheric vector magnetogram observations have shown that the opposite polarities of a long arcade structure move apart and then come together. In addition to this "sliding doors" effect, orientations of horizontal magnetic fields along the polarity inversion line on the photosphere evolve from a normal-polarity configuration to an inverse one. To explain this behavior, a simple model by Okamoto et al. suggested that it is the result of the emergence of a twisted flux rope. Here, we model this scenario using a three-dimensional megnatohydrodynamic simulation of a twisted flux rope emerging into a pre-existing overlying arcade. We construct magnetograms from the simulation and compare them with the observations. The model produces the two signatures mentioned above. However, the cause of the "sliding doors" effect differs from the previous model.
\end{abstract}

Key words: magnetohydrodynamics (MHD) - Sun: photosphere

Online-only material: color figures

\section{INTRODUCTION}

Recent observations (Okamoto et al. 2008, 2009, hereafter referred to as $\mathrm{O} 1$ and $\mathrm{O} 2$, respectively) of the region NOAA AR 10953 with the Solar Optical Telescope (SOT) on board the Hinode satellite have revealed interesting behavior. Part of this region contains a prominence lying along the polarity inversion line (PIL) of a magnetic arcade. $\mathrm{O} 1$ and $\mathrm{O} 2$ suggest that the prominence is formed/maintained by the full emergence of a twisted flux rope along the PIL. This idea is based on two main signatures that are observed in photospheric vector magnetograms. The first is the "sliding doors" effect where the opposite polarities of the arcade appear to expand laterally a certain distance before beginning to narrow. The second signature is that the orientation of horizontal magnetic fields at the PIL changes from a normal-polarity configuration to an inverse one, i.e., vectors pointing across the PIL in one direction gradually change to pointing in the other direction.

In this Letter, we test the above idea by constructing a threedimensional magnetohydrodynamic (3D MHD) simulation of a twisted flux rope emerging into an overlying arcade. Here, we focus on activity at the base of the photosphere and leave consideration of the response of the upper atmosphere to future work.

Previous studies have considered the emergence of a twisted flux rope into a magnetic arcade. For example, Fan \& Gibson (2004) consider a "kinematic" flux emergence model. Here, the emergence is driven by prescribing a time variation of the electric field on the lower boundary of the box. The main focus of their study is coronal mass ejection generation.

We consider a dynamic, self-consistent flux emergence model, where the top of the solar interior is included and the flux rope is left to rise and emerge through buoyancy effects. This allows the dynamics at the base of the photosphere to be studied without artificially imposed flows.

\section{MODEL}

The 3D compressible and resistive MHD equations are solved using a Lagrangian remap scheme (Arber et al. 2001). In dimensionless form, these are

$$
\begin{gathered}
\frac{\partial \rho}{\partial t}+\nabla \cdot(\rho \mathbf{u})=0, \\
\rho\left(\frac{\partial \mathbf{u}}{\partial t}+(\mathbf{u} \cdot \nabla) \mathbf{u}\right)=-\nabla p+(\nabla \times \mathbf{B}) \times \mathbf{B}+\nabla \cdot \mathbf{T}+\rho \mathbf{g}, \\
\frac{\partial \mathbf{B}}{\partial t}=\nabla \times(\mathbf{u} \times \mathbf{B})+\eta \nabla^{2} \mathbf{B}, \\
\rho\left(\frac{\partial \varepsilon}{\partial t}+(\mathbf{u} \cdot \nabla) \varepsilon\right)=-p \nabla \cdot \mathbf{u}+\eta j^{2}+Q_{\text {visc }}, \\
\nabla \cdot \mathbf{B}=0,
\end{gathered}
$$

with specific energy density

$$
\varepsilon=\frac{p}{(\gamma-1) \rho} .
$$

The basic variables are the density $\rho$, the pressure $p$, the magnetic field vector $\mathbf{B}$, and the velocity vector $\mathbf{u} . j$ is the magnitude of current density and $\mathbf{g}$ is the gravity (uniform in the $z$-direction). $\gamma(=5 / 3)$ is the ratio of specific heats and $\eta$ is the uniform resistivity. The variables are made dimensionless against photospheric values, namely, pressure, $p_{\mathrm{ph}}=1.4 \times$ $10^{4} \mathrm{~Pa}$; density, $\rho_{\mathrm{ph}}=3 \times 10^{-4} \mathrm{~kg} \mathrm{~m}^{-3}$, and scale height $H_{\mathrm{ph}}=170 \mathrm{~km}$. The other units used in the simulations are temperature, $T_{\mathrm{ph}}=p_{\mathrm{ph}} /\left(R \rho_{\mathrm{ph}}\right)=5.6 \times 10^{3} \mathrm{~K}$; speed, $u_{\mathrm{ph}}=\left(p_{\mathrm{ph}} / \rho_{\mathrm{ph}}\right)^{1 / 2}=6.8 \mathrm{~km} \mathrm{~s}^{-1} ;$ time, $t_{\mathrm{ph}}=H_{\mathrm{ph}} / u_{\mathrm{ph}}=25 \mathrm{~s}$; and magnetic field $B_{\mathrm{ph}}=\left(2 \mu_{0} p_{\mathrm{ph}}\right)^{1 / 2}=1.3 \times 10^{3} \mathrm{G}$. We take $\eta=0.001$. The viscosity tensor and viscous contribution to the energy equation are, respectively,

$\mathbf{T}=\mu\left(\nabla \mathbf{u}+\nabla \mathbf{u}^{\mathrm{T}}-\frac{2}{3} \mathbf{I} \nabla \cdot \mathbf{u}\right), \quad Q_{\text {visc }}=\mathbf{T}: \frac{1}{2}\left(\nabla \mathbf{u}+\nabla \mathbf{u}^{\mathrm{T}}\right)$,

where $\mu$ is the viscosity and $\mathbf{I}$ is the identity tensor. We use actual viscosity to aid the relaxation of the equilibrium and take $\mu=0.001$.

For the initial condition, we model the solar atmosphere as a plane-parallel stratified layer with a solar interior (marginally stable to convection), an isothermal photosphere/ chromosphere, a transition region, and an isothermal corona. 


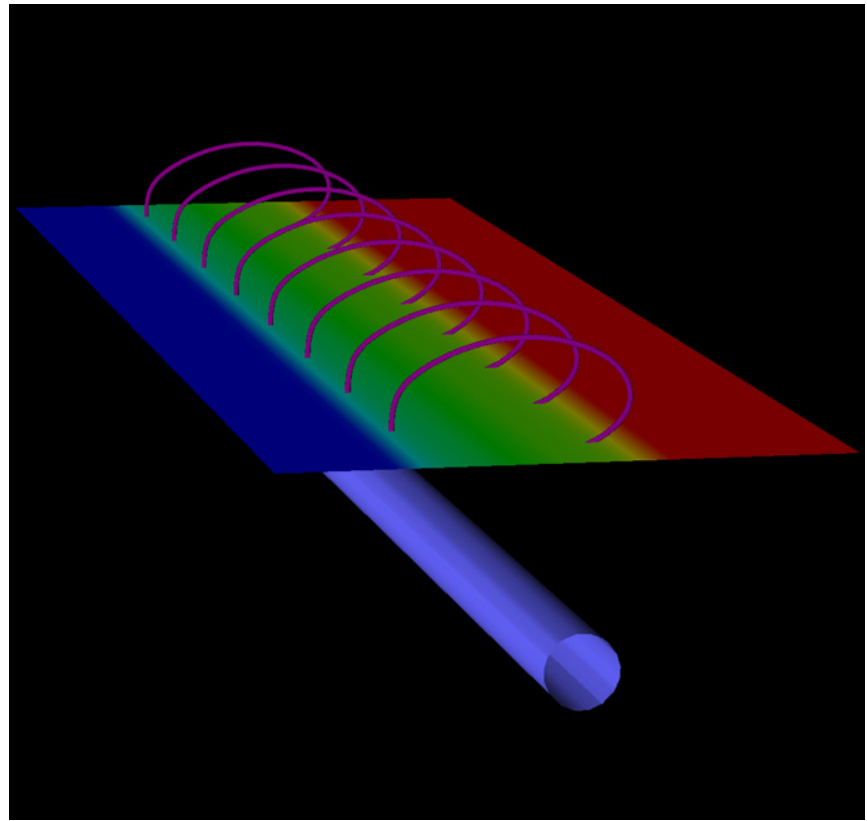

Figure 1. Initial condition. The magnetogram of $B_{z}$ at the base of the photosphere shows the opposite polarities of the arcade (blue: negative, red: positive, and green: horizontal field). Some of the arcade field lines are traced in purple from the base of the photosphere. An isosurface $(|\mathbf{B}|=3)$ shows the initial location of the twisted flux rope in the solar interior.

(A color version of this figure is available in the online journal.)

The stratification is identical to that in MacTaggart \& Hood (2009a). A numerical equilibrium with an arcade is constructed from the interaction and relaxation of magnetic fields (e.g., MacTaggart \& Hood 2009b). With the equilibrium setup, a uniformly twisted magnetic cylinder is placed in the solar interior. We choose a weakly twisted flux tube of $1 /(10 \pi)$ turns per unit length of the axis. The orientation of the twist is chosen to inhibit reconnection with the overlying arcade, i.e., when the tube comes into contact with the arcade, their respective fields are not antiparallel. The tube is made buoyant with respect to its surrounding plasma in the solar interior. To encourage an $\Omega$-loop to form, the density deficit is multiplied by an exponential function of the form $\exp \left(-y^{2} / \lambda^{2}\right)$, where $y$ is the distance along the tube axis and $\lambda$ is an adjustable parameter (e.g., Fan 2001). In this Letter, we take $\lambda=40$. This is a large value (compared with previous simulations) in order to model the emergence of a long tube. If there was no kink in the tube (i.e., $\lambda=\infty$ ) then the flux rope axis would not rise above the base of the photosphere in this model. A visualization of the initial condition is displayed in Figure 1. The basic processes of the emergence of flux tubes have been discussed in many previous works (e.g., Archontis et al. 2004; Leake \& Arber 2006; Murray et al. 2006; MacTaggart \& Hood 2009b).

The flux rope in the solar interior is initially placed at $(x, 0, z)=(0,0,-15)$, which has an axial field strength of $|\mathbf{B}|=8$ and a radius of 2.5 . When the tube rises to just below the photosphere and begins to interact with the arcade, its field strength has reduced to approximately $3 / 2$ times that of the arcade field strength.

\section{RESULTS}

\subsection{First Signature- "Sliding Doors"}

In the model of $\mathrm{O} 1$, the "sliding doors" effect is produced by the lateral motions of the arcade polarities as a twisted flux rope passes through the base of the photosphere as a "solid" cylinder. The "sliding doors" effect is also present in our model; however, the mechanism behind it is different. One important aspect which is not present in the model of $\mathrm{O} 1$ is that the emerging flux rope has a $B_{z}$ profile of its own and so will have an imprint on the magnetograms. In our model, the "sliding doors" effect is produced by the rise, expansion, and emergence of the flux tube. Figure 2 displays slices in the $(x, 0, z)$ plane at four different times. Each slice portrays regions of $B_{z}$ (colors and contours) and $\left(B_{x}, B_{z}\right)$ arrows to indicate the position of the tube axis.

The first phase of the "sliding doors" effect is the rise of the flux tube to the photosphere. In the solar interior, the flux tube follows a field-free path. It only comes into contact with the arcade, which is anchored in the solar interior, just below the photosphere. As the flux tube rises, it expands. Due to its dominant pressure, the flux tube pushes the opposite polarities of the overlying arcade apart. In the cartoon model, this would be in the broadening phase of the "sliding doors" effect. However, this is not the case in our model as the flux tube continues to push upward and so contributes to the $B_{z}$ map seen on magnetograms. Figure 2(a) shows the interaction of the flux rope with the overlying arcade at $t=38$. Here, the tube has pushed the arcade aside, and its own magnetic field has risen above $z=0$ and into the photosphere. The tube axis is still below $z=0$.

The second phase of the "sliding doors" effect is the lateral expansion of the tube in the photosphere. Since the photosphere is stable to buoyancy, the flux rope cannot continue rising due to buoyancy (the presence of the top of the flux tube in the photospheric layer is due to momentum carrying it there). Instead it expands laterally. Figure 2(b) shows this expansion at $t=48$. The contours in Figure 2(b) are further apart than those in Figure 2(a). Hence, the region of weak $B_{z}$ at the center of the emerging tube (and hence also at the PIL) is broader. This broadening is the first part of the "sliding doors" effect. Figure 2(b) also shows that the axis of the flux tube is now above the base of the photosphere $z=0$. Although, as mentioned before, the photosphere is stable to buoyancy, the magnetic field from the solar interior still rises upward and can push through $z=0$.

With the continued lateral expansion in the photosphere combined with the buoyant rise of flux from below, the emerging flux tube eventually becomes subject to the magnetic buoyancy instability. Figure 2(c) displays the initial stage of this at $t=58$. Here, the two main regions of $B_{z}$ have risen upward. As the field emerges into the atmosphere, the $B_{z}$ profile becomes stronger in the central region (at the PIL). Figure 2(d) shows the emergence at the later time of $t=78$. Now the tube's field has penetrated far into the atmosphere, and the contours of $B_{z}$ near the base of the photosphere $(z=0)$ have moved closer together. This narrowing is the second part of the "sliding doors" effect. A time slice of this broadening and narrowing at $z=0$ is shown in Figure 3.

In short, the "sliding doors" effect is produced from the expansion of the flux rope at the photosphere and its subsequent emergence, via the magnetic buoyancy instability, into the atmosphere. Note that the axis of the flux tube does not continue rising but becomes trapped at a height of $z \approx 1$.

\subsection{Second Signature-Horizontal Vector Directions}

In principle, it is possible to explain the broadening and narrowing motions discussed in the previous section without recourse to flux tube emergence. However, this would be to 


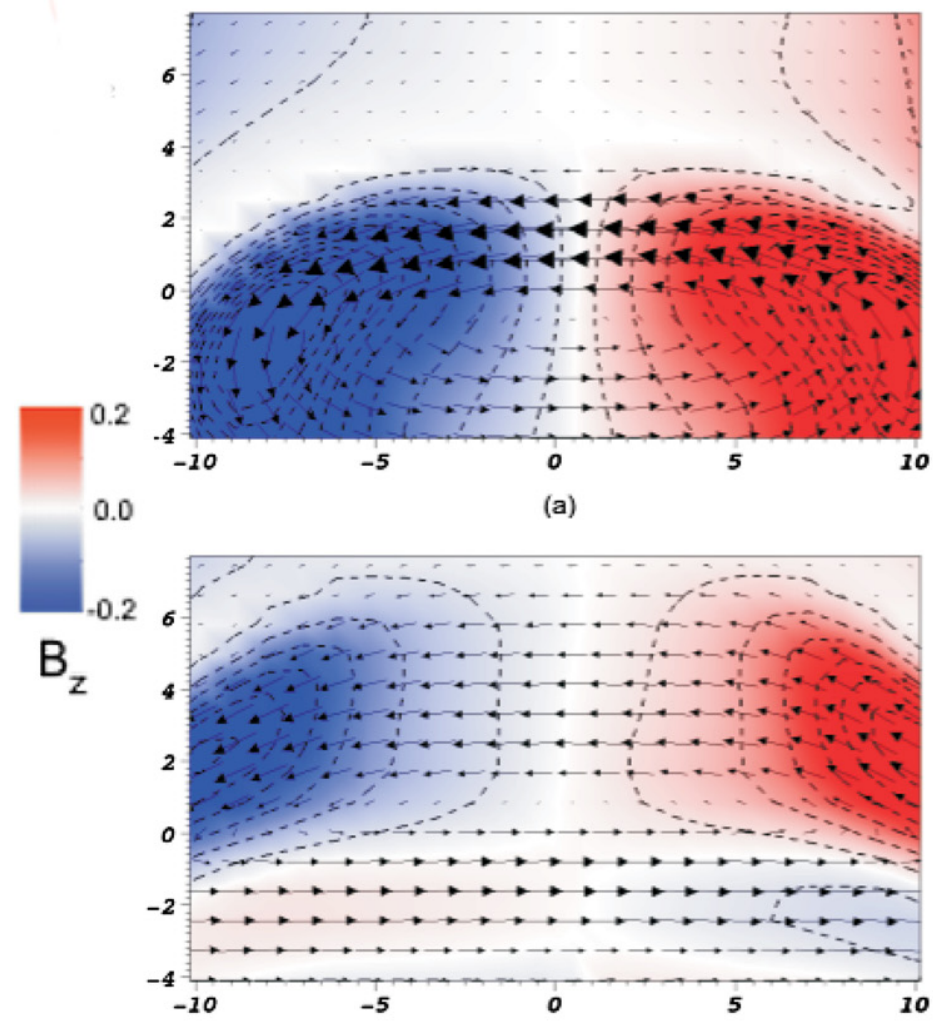

(c)

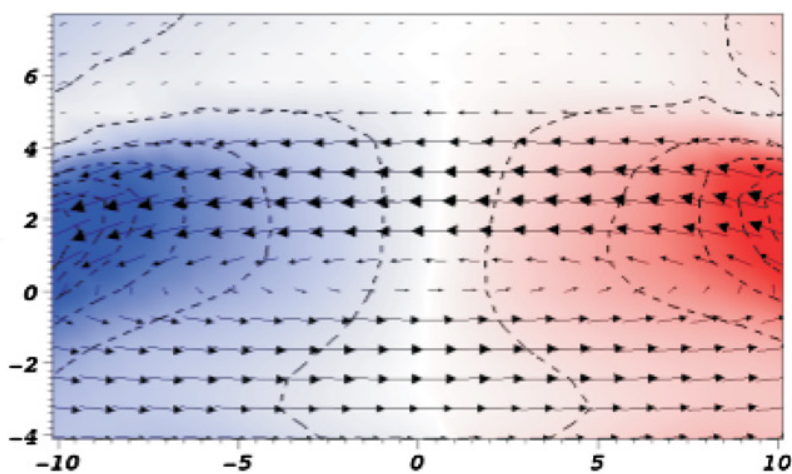

(b)

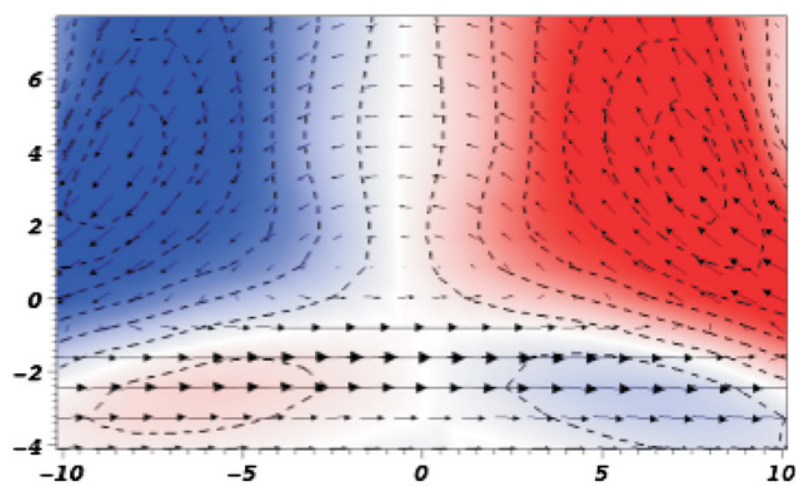

(d)

Figure 2. Slices in the $(x, 0, z)$ plane. Dashed contours and colors identify regions of strong (relative) $B_{z} .\left(B_{x}, B_{z}\right)$ arrows indicate the position of the flux tube axis. Each slice represents a different time: (a) $t=38$, (b) $t=48$, (c) $t=58$, and (d) $t=78$.

(A color version of this figure is available in the online journal.)

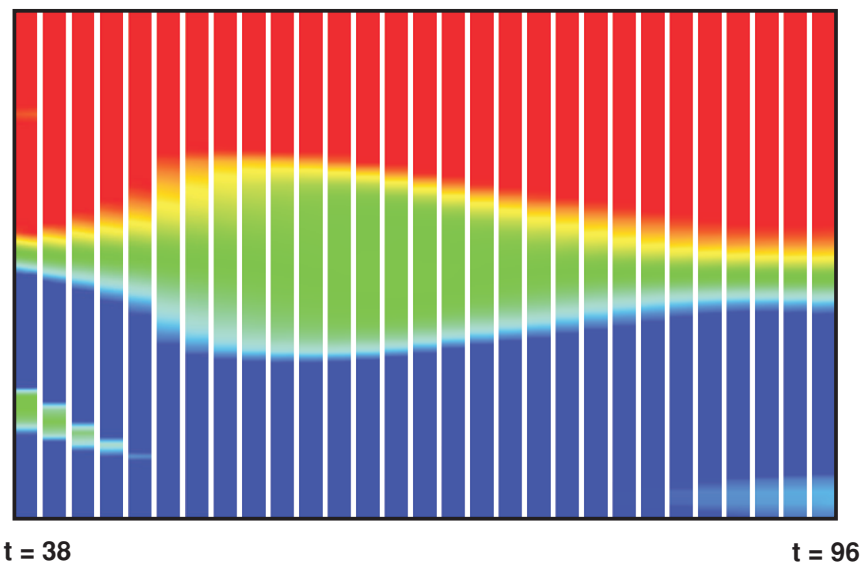

Figure 3. Time-slice image showing the "sliding doors" effect. The slices are taken at $y=0$ and from $x=-20$ to $x=20$. The time difference between the slices is 2 in non-dimensional units and should be compared with Figure 1(d) of O2. Colors are as indicated in Figure 1.

(A color version of this figure is available in the online journal.)

neglect the second signature of the change in the orientation of the horizontal photospheric magnetic field vectors. Figure 4 shows the time evolution of the angle of the horizontal field against the PIL for two positions along the tube length. The graph should be read such that if the PIL faces north $\left(0^{\circ}\right)$, west is $-90^{\circ}$ and east is $90^{\circ}$.

Due to the large value chosen for $\lambda$, the $\Omega$-shape is not greatly pronounced. This can account for the similar profiles of the two curves in Figure 4. However, as mentioned earlier, the kink is

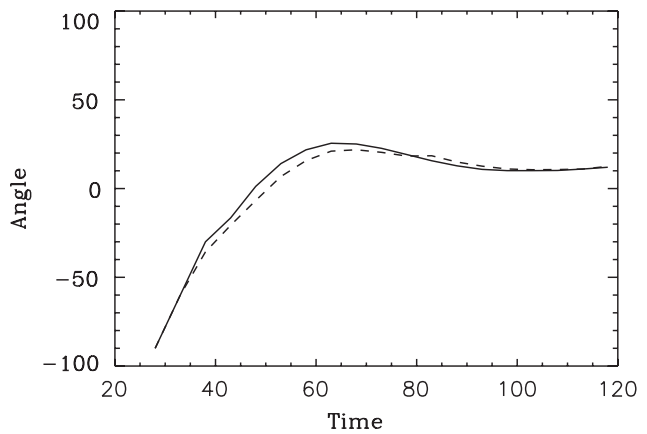

Figure 4. Time evolution of the horizontal field vector against the PIL for two locations along the tube length. Key: $y=0$, solid; $y=10$, dash.

necessary for the drainage of plasma and, hence, the rise of the flux tube axis above the base of the photosphere. Figure 4 clearly shows the switch in vector direction from one side of the PIL to the other. Both curves move through $90^{\circ}$ and then reach a maximum angle on the other side of the PIL before appearing to settle at a smaller, but positive, angle. This information is presented in $\mathrm{O} 1$ in Figure 2, panels (d1)-(d6). Although the angles at various positions along the PIL vary due to convection, it could be argued that for large sections of the observed PIL, the angles follow a profile similar to that in Figure 4. This rotation of the horizontal field vector does require the axis to rise above $z=0$, even though it is only by a small amount, i.e., the axis does not rise to coronal heights. The top of the tube, however, is able to reach coronal heights due to the magnetic buoyancy instability. 


\section{DISCUSSION}

In the studies of $\mathrm{O} 1$ and $\mathrm{O} 2$, they put forward two main reasons why they believe it is the emergence of a twisted flux rope into the overlying arcade that takes place. The first is the broadening and subsequent narrowing of the opposing polarities of the arcade observed on magnetograms - the "sliding doors" effect. The second is that the horizontal magnetic field at the PIL is observed to gradually change polarity. To study this scenario, we have constructed a dynamic flux emergence model with an overlying magnetic arcade field. Our model is in good agreement with the second signature since it represents the rising of the axis above the plane where the magnetograms are taken (at the base of the photosphere, $z=0$, in our model). The "sliding doors" effect is also present but its cause is different to that previously suggested. When the flux tube rises to the photosphere it has a $B_{z}$ profile of its own. As the tube expands at the photosphere, its opposite polarities of $B_{z}$ broaden. When the tube becomes unstable to the magnetic buoyancy instability, the $B_{z}$ profile strengthens at the PIL and so the opposite polarities appear to narrow. The "sliding doors" effect is then produced in our model by the emergence of the flux tube field rather than the lateral motions of the overlying arcade.

We believe that the findings of this work will still hold after the inclusion of extra physics. One simplification of the model is to treat the photosphere and lower chromosphere as fully ionized. In reality, the resistivity is increased in this region due to partial ionization (Cowling 1957; Leake \& Arber 2006; Arber et al. 2007). As a simple test, we perform the simulation with an increased resistivity of $\eta=0.004$. Although there is increased magnetic diffusion, the two signatures and their underlying mechanisms remain, qualitatively, unchanged.
Another simplifying assumption is that of a solar interior which is marginally stable to convection. In $\mathrm{O} 1$ and $\mathrm{O} 2$, the scale of the "sliding doors" effect is much larger than the granular scale. Also, from flux emergence simulations including radiative transfer and convection (e.g., Martinez-Sykora et al. 2008), the emerging tube deforms the convection, elongating the cells. It is the active region magnetic field which plays a dominant role in determining the photospheric velocity field.

D.M. acknowledges financial assistance from STFC. The computational work for this Letter was carried out on the joint STFC and SFC (SRIF) funded cluster at the University of St. Andrews. D.M. and A.W.H. acknowledge financial support form the European Commission through the SOLAIRE Network (MTRN-CT-2006-035484). D.M. and A.W.H. also thank those who organized and participated in the MSSL flux emergence workshop. It was this workshop that provided the impetus for this Letter.

\section{REFERENCES}

Arber, T. D., et al. 2001, J. Comput. Phys., 171, 151

Arber, T. D., et al. 2007, ApJ, 666, 541

Archontis, V., et al. 2004, A\&A, 426, 1047

Cowling, T. G. 1957, Magnetohydrodynamics (New York: Interscience)

Fan, Y. 2001, ApJ, 554, L111

Fan, Y., \& Gibson, S. E. 2004, ApJ, 609, 1123

Leake, J. E., \& Arber, T. D. 2006, A\&A, 450, 805

MacTaggart, D., \& Hood, A. W. 2009a, A\&A, 507, 995

MacTaggart, D., \& Hood, A. W. 2009b, A\&A, 501, 761

Martinez-Sykora, J., et al. 2008, ApJ, 679, 871

Murray, M. J., et al. 2006, A\&A, 460, 909

Okamoto, T. J., et al. 2008, ApJ, 673, L215

Okamoto, T. J., et al. 2009, ApJ, 697, 913 